\title{
La universidad como herramienta de crecimiento y cambio
}

\section{The university as a tool for growth and change}

\section{Karina Silva}

Facultad de Humanidades y Ciencias de la Educación Universidad de la República mag.silva.garcia.karina@gmail.com

Recebido em 31/08/2017

Aceito em 10/09/2017

Para citar este artigo:

\section{Resumen}

A lo largo de las ultimas décadas hemos sido testigos de la expansión de la economía con base en el conocimiento y la consecuente consolidación de las sociedades de conocimiento. Una de las consecuencias más notorias de esta situación es el reconocimiento de la universidad como institución responsable de colaborar con el desarrollo económico y crecimiento social. Ante esta nueva realidad, las universidades de distintos países realizaron modificaciones más o menos profundas y en períodos de tiempo más o menos extensos. A nivel regional, estas transformaciones han sido muy distintas y se han visto dificultadas por factores sociales vinculados a la distribución de las riquezas y la democratización del conocimiento.

En este articulo buscamos mostrar, primero, la pertinencia de una inminente transformación de la universidad de Latinoamérica para alcanzar mejores niveles de cohesión social, crecimiento y desarrollo científico-tecnológico. Segundo, procuramos evidenciar la utilidad de acudir a experiencias de otros países en materia de transferencia de conocimiento como tercera misión de la universidad contemporánea y a la elaboración políticas científicas para promover el desarrollo local.

Palabras clave: Universidad, Transferencia, Desarrollo, Cohesión social

\section{Abstract}

Throughout the last decades we have witnessed the expansion of the knowledge based economy and the consequent consolidation of knowledge societies. One of the most striking consequences of this situation is the recognition of the university as an institution responsible for collaborating in economic development and social growth. Faced with this new reality, universities in different countries have made deeper or less deep changes in more or less extensive periods of time. At regional level, these transformations have been very different and hampered by social factors linked to wealth distribution and knowledge democratization.

In this article, we intend to show, firstly, the pertinence of an imminent transformation of the university in Latin America in order to achieve better levels of social cohesion, growth and scientific-technological development. Secondly, we wish to demonstrate the usefulness of applying other countries' experiences in the field of knowledge transfer as the third mission of the contemporary university and developing scientific policies to promote local development.

Key-words: University, Transfer, Development, Social cohesion 


\section{La universidad iberoamericana: una realidad compleja y desafiante}

Iberoamérica alberga entre sus países las más diversas realidades, no obstante, todos los países iberoamericanos tienen el objetivo común de alcanzar grados favorables de cohesión social y ciudadanía que les permitan incorporarse correctamente a la sociedad y a la economía de conocimiento. Cohesión social y ciudadanía, sostiene Mario Albornoz, son "el resultado de dos miradas complementarias sobre un mismo fenómeno: la constitución de sociedades integradas e integradoras de ciudadanos que libremente asumen su pertenencia a ella" (Albornoz, M., 2012). La cohesión social es una característica de aquellos países cuyos ciudadanos tienen posibilidades reales de ser incluidos como ciudadanos de derecho. Esta inclusión supone que los ciudadanos cuentan con una equitativa distribución de las riquezas, acceso a prestaciones sociales, igualdad de oportunidades para el desarrollo personal, ejercicio de derechos, cumplimiento de deberes y participación real como miembros de una sociedad. La ciencia, sostiene Albornoz, pasa a ser un elemento clave en las sociedades con cultura de cohesión social en las que los ciudadanos pueden acudir a la ciencia por dos motivos o fines generales. Primero, los ciudadanos pueden acudir tanto a las ciencias sociales como a las ciencias naturales en busca de una mirada crítica del entorno social y/o natural que los rodea. Segundo, pueden acudir a la ciencia para la satisfacción de una necesidad. Por lo tanto, la cohesión social requiere tanto de la ciencia y la tecnología como de la cultura científica; esto supone una mayor comprensión de la ciencia, la tecnología y la innovación y de los procesos vinculados a estos; colabora, así, con una mayor cohesión social. Tal como lo sostiene el autor, "la ciencia, la tecnología y la innovación pueden contribuir a mejorar la capacidad institucional de Estado y los mecanismos de reproducción de la sociedad civil, mejorando la cohesión social" (Ídem).

Al igual que la mayor parte de los países en vías de desarrollo, en los países iberoamericanos se observa una clara desigualdad en la distribución de riquezas y en el acceso al conocimiento tanto entre países como entre personas. En la Cumbre de Jefes de Estado y Gobierno de los países iberoamericanos celebrada en Mar del Plata, Argentina, en 2010, se estableció el programa Metas Educativas 2021 mediante el cual se procura transformar la educación para alcanzar sociedades mejor formadas y más ambiciosas. Para cumplir con ello, la principal transformación debe ser llevada a cabo en todos los niveles de la educación, pero fundamentalmente en la educación universitaria y profesional. Se debe apostar a la universalización de la educación; promover y desarrollar una educación sensible a los cambios tecnológicos y a las nuevas formas de desarrollo de conocimiento científico y de innovación. Consideradas en conjunto, estas modificaciones permitirían alcanzar un desarrollo económico equilibrado que permita reducir la pobreza y la desigualdad y alcanzar un nivel positivo de cohesión social (Marchesí, A., 2012, en Albornoz, M.; López Cerezo, J. A. (ed.), 2012). En suma, se reconoce la importancia de incrementar la cultura científica de la ciudadanía como uno de los caminos posibles que favorezcan el cambio.

Siguiendo a José A. López Cerezo, cuando hablamos de cultura científica nos referimos al conocimiento científico básico con el que cuenta una sociedad, así como a la conciencia crítica sobre las potencialidades y limitaciones de la ciencia y la adopción de pautas de comportamiento por parte de los actores sociales para la toma de decisiones sobre el desarrollo del conocimiento científico-tecnológico. Por lo que la educación, en tanto herramienta transformadora y habilitadora de una nueva realidad en Iberoamérica, debe ser tanto científica como cívica. Es decir, la educación científica no solo debe ser propedéutica, sino que además debe promover el desarrollo de ciudadanos que se involucren en la vida pública sobretodo en materia de ciencia y tecnología.

Además de este proyecto común, en las últimas décadas los gobiernos iberoamericanos han adquirido por separado cierta sensibilidad en lo que refiere a la importancia de la ciencia, la tecnología y la innovación para el desarrollo (López Cerezo, J. A.; Cámara Hurtado, M., 2012 en Albornoz, M., López Cerezo, J. A. (ed.), 2012). Esto motivó la elaboración de políticas específicas que buscan incrementar el desarrollo del conocimiento científico-tecnológico y la innovación, así como la cultura científica de la población (Albornoz, M.; López Cerezo, J. A., 2012). Por lo tanto, parece ser útil abordar de modo crítico las relaciones que se dan entre ciencia, tecnología y sociedad para alcanzar un modelo que conduzca al desarrollo social anhelado. Esta tarea bien puede ser considerada homérica, aunque no por ello debe ser desestimada. 


\section{Reconociendo la heterogeneidad}

Dadas las diferencias estructurales entre los países que integran el espacio iberoamericano, es imposible establecer algo así como un único camino hacia la modernización y alcanzar los estándares internacionales de desarrollo. Afirmar lo contrario supone, tal como sostienen Albornoz y López Cerezo, una visión reduccionista del ámbito social y una visión benéfica de la innovación, lo que nos llevaría a un reduccionismo tecnocrático de la gestión de políticas científicas. En efecto, tal como sostiene Albornoz, las sociedades iberoamericanas presentan una estructura social desigual, motivo por el cual "los parámetros de una política científica y tecnológica basados en la repetición mimética de enfoques que son empleados en países con mayor grado de desarrollo configuran un camino sin salida y pueden convertirse en una frivolidad intelectual" (Albornoz, M., 2012 en Albornoz, M., López Cerezo, J. A. (ed.), 2012). Tal como lo ha propuesto John Ziman, la ciencia forma parte de la sociedad y no al revés y por lo tanto son las características de la sociedad las que establecen las condiciones de posibilidad para el funcionamiento de un modelo particular de investigación y desarrollo científico-tecnológico (Ziman, J., 2003). Por otra parte, Hebe Vessuri sostiene al respecto que las transformaciones sociales vinculadas a la ciencia, la tecnología y la innovación son contingentes y estas se dan por un conjunto de variables presentes en la configuración social en los que influyen, entre otras cosas, la herencia histórica. En palabras de la autora,

Los procesos de cambio tienen lugar en diferentes ámbitos y arrastran historias particulares, conduciendo a resultados diferentes que se perfilan en variadas configuraciones organizacionales. No se resumen en un único proceso de cambio, sino que funcionan de acuerdo con sus distintos legados históricos, cuya interacción precisa considerarse cuidadosamente, pues los logros suelen ser contingentes y variables (Vessuri, H., 2014: 170).

Consecuentemente, siguiendo nuevamente a Albornoz, si bien la ciencia es una herramienta imprescindible para que las sociedades superen muchas de sus dificultades, no es posible imponer modelos o senderos pre-formateados.

En definitiva, la experiencia de las últimas décadas conlleva a la percepción de que existen riesgos asociados a determinados patrones de desarrollo científico y tecnológico. Se configura así un horizonte dicotómico de oportunidades y amenazas sobre las que las sociedades deben pronunciarse y aprender a gestionarse mediante adecuadas políticas de educación, ciencia y tecnología (Albornoz, M., 2012 en Albornoz, M., López Cerezo, J. A. (ed.), 2012).

Por lo tanto, si bien la ciencia es un instrumento de transformación social, no todo modelo de desarrollo y gestión de la investigación científico-tecnológica funciona correctamente en cualquier país. Por lo que, si bien es posible y hasta deseable analizar los distintos estilos de $\mathrm{I}+\mathrm{D}+\mathrm{i}$, no es posible realizar una transferencia de modelos implementados en otros países. Es necesario reconocer la heterogeneidad tanto local como de la región y en base a ello elaborar políticas, programas y proyectos de cooperación que colaboren con revertir la disparidad distributiva y la exclusión social. En este camino, es necesario reconsiderar los modelos educativos y de investigación científica, apostando a aquellos diseños que promuevan la heterogeneidad y cooperación social entre los actores del país primero y, de la región después. Tal como sostienen Albornoz y López Cerezo, es necesario

favorecer las capacidades básicas de los recursos humanos y la creación de centros de referencia, promover la vinculación sistémica de las instituciones de ciencia y tecnología con las demandas sociales y estimular la difusión social del conocimiento y la participación ciudadana, y orientar políticas de investigación y desarrollo $(\mathrm{I}+\mathrm{D})$ hacia una convergencia con las políticas sociales (Albornoz, M.; López Cerezo, J. A., 2012). 
No se trata de negar las diferencias sino reconocerlas y desarrollar conforme a esta diversidad, una gobernanza de los sistemas de ciencia y tecnología. El desafío consiste en lograr una articulación entre el sistema de producción de conocimiento científico-tecnológico e innovación y las demandas sociales. Tal como sostiene Vessuri, "Los desafíos actuales están estrechamente conectados a la aceleración de la producción y el consumo, el crecimiento poblacional, la globalización socioeconómica y cultural, y patrones difundidos de desigualdad" (Vessuri, H., 2014: 172). Es decir, el desafío consiste en desarrollar sistemas de producción de conocimiento que estén al servicio de la ciudadanía (Tiana Ferrer, A., 2012 en Albornoz, M., López Cerezo, J. A. (ed.), 2012). Para ello, señala Albornoz, es necesario incrementar las inversiones locales en I+D y proveer de mecanismos que permitan una mayor utilización de los conocimiento generados localmente mediante la articulación de los diversos actores y sectores sociales, tanto locales como regionales.

En el año 2006 la Conferencia latinoamericana y del Caribe sobre Ciencia, Tecnología e Innovación para el Desarrollo Sostenible realizada por la UNESCO, señaló que uno de los principales problemas de los países latinoamericanos era la falta de articulación entre los actores nacionales y la carencia de mecanismos de integración regional. En esta misma conferencia se señaló cierto desajuste entre las esferas de producción y uso del conocimiento que se traduce en un desencuentro entre la investigación académica y las empresas con respecto a las expectativas del conocimiento producido ${ }^{1}$. Asimismo, se señaló que estos países presentaban un claro desajuste en la órbita de la democratización y la satisfacción de necesidades sociales.

En efecto, señala Albornoz, entre los problemas que aun tienen por delante los países iberoamericanos es hacer que la investigación científica sea relevante según intereses sociales (Albornoz, M., 2012 en Albornoz, M., López Cerezo, J. A. (ed.), 2012). Por lo tanto, siguiendo la propuesta de este autor, los estados tienen la difícil tarea de diseñar políticas públicas que atiendan a los procesos de producción, difusión, transferencia y uso del conocimiento. Para cumplir con esto, es necesario repensar modelos educativos y de investigación que reconozcan y asuman la heterogeneidad de la realidad en la que se desarrollan. Al mismo tiempo, es necesario pensar y diseñar organizaciones redes que permitan la fluida comunicación entre especialistas, políticos, ciudadanos, empresarios, productores, etcétera, etcétera.

En los últimos años, señala Albornoz, en el mundo hay una tendencia creciente -aunque no homogénea, a realizar cambios institucionales y legales orientados a la articulación interna y externa de los sistemas de innovación. Si bien se apuesta a la cooperación y al diseño de estrategias locales, no todo sistema de innovación es socialmente aceptable. El diseño de políticas de ciencia y tecnología debe tener en cuenta esto y promover un modelo de sistema social que logre articular todos los vértices mediante el trabajo con todos los actores sociales y no solo con la comunidad de expertos. Para cumplir con esta difícil tarea, tal como lo sostuvo la CEPAL en el año 2007, el aporte que las Ciencias Sociales en general, es colaborar en la reflexión crítica de posibles modelos a ser implementados y en el diseño de indicadores que permitan monitorear el éxito o fracaso de las políticas públicas planteadas para alcanzar o mejorar la cohesión social (Ídem).

Pero la exigencia de la relevancia social de la investigación científica no es una novedad sino que es un problema ante el que se enfrentan tanto los que toman decisiones políticas sobre la ciencia como aquellos que toman a la ciencia y la tecnología como objeto de estudio. Propuestas como las de Michael Gibbons (Gibbons, M., et al., 1994) y de Henry Etzkowitz (Etzkowitz, H., 1998) son, en efecto, modelos que refieren a la producción de conocimiento socialmente relevante. Más allá de las dificultades que presentan estos modelos y las críticas que pueden realizarse al respecto de lo que proponen, en ambos casos se promueve un modo de entretejer a la ciencia, el estado, el mercado y la sociedad civil, en donde la universidad es la encargada de proveer de conocimiento nuevo. La originalidad de estas perspectivas, señala Albornoz, no está en la visión utilitarista que puede rastrearse incluso hasta Francis Bacon. "El matiz que se añade en estas nuevas formulaciones es el de la existencia de una trama social que no solamente asigna utilidad ex post, sino que asume algún grado de protagonismo en todo el proceso de gestación y despliegue de la investigación hacia 
su efectiva utilización" (Albornoz, M., 2012). En este sentido, entendemos que las propuestas de Gibbons y Etzkowitz pueden ser consideradas como modelos a partir de los cuales establecer mecanismos que permitan hacer que la investigación científico-tecnológica adquiera relevancia social y así encarar el principal desafío de los países Iberoamericanos y, en particular, el más importante de los problemas de los países de América Latina en los que la universidad tiene un rol sustancial.

Desde nuestro punto de vista, el resultado substancial de analizar los sistemas de producción y gestión de conocimiento científico-tecnológico a la luz de estos modelos es que queda en evidencia que no es suficiente el desarrollo de políticas de innovación. Esto no quiere decir, tal como lo sostienen Elena CastroMartínez y Judith Sutz, que no sea posible desarrollar políticas de innovación eficaces, "sino que exigen una creatividad sostenida, una gran vocación de aprender de otras experiencias y una no menor vocación para analizar cuidadosamente qué de estos últimos puede ser adoptado y adaptado" (Castro-Martínez, E.; Sutz, J., 2012, en Albornoz, M.; López Cerezo, J. A. (ed.), 2012). Es necesario, sostienen estas autoras, estudiar las particularidades de las sociedades, identificar potencialidades, defectos y virtudes $y$, a partir de ello, diseñar modelos que apuesten a mejorar la situación.

\section{La universidad como elemento de transformación}

Las transformaciones que ha sufrido la universidad en todo el mundo, y en Iberoamérica en particular, son algunas de las modificaciones que se han llevado a cabo en el último siglo para atender las exigencias de las nuevas sociedades. Esta transformación es consecuencia directa del reconocimiento de la universidad como la institución por excelencia dedicada a la producción de conocimiento nuevo y socialmente útil. Surge así la tercera misión de la universidad, con el objetivo de estrechar la distancias entre la producción del conocimiento científico-tecnológico y sus potenciales usuarios. En todo el mundo se han diseñado y puesto en práctica distintas estructuras para impulsar y gestionar los vínculos entre la universidad y el sector productivo y los ciudadanos en general con la convicción de que ello colaborará con una mejora en los niveles de desarrollo locales.

Tradicionalmente, sostiene Vessuri, las universidades en Latinoamérica, en particular, se han caracterizado por ser dispensadoras de títulos más que proveedoras de conocimiento nuevo. La masificación en la matrícula de estudiantes en las décadas de los ochenta y los noventa ha colaborado con que esto se mantenga. No obstante, con la intención de revertir esta tendencia y de atender a las demandas sociales inmediatas, se ha estimulado el desarrollo de alianzas tanto en el interior de la universidad como fuera de esta. Se apostó, fundamentalmente, a mejorar el vínculo entre la universidad y el sector productivo mediante la consolidación de parques tecnológicos, incubadoras de empresas, empresas universitarias, entre otras. En efecto, "los diferentes gobiernos nacionales y estatales se muestran cada vez más interesados en estimular esta cooperación, entre otras cosas por considerarla una ruta plausible para el desarrollo regional" (Vessuri, H., 1997: 137). Tradicionalmente, el propósito de la universidad fue producir conocimiento por el valor del conocimiento mismo; en la actualidad el propósito que se demanda de la universidad es de carácter pragmático, y se vincula estrechamente con los sectores industrial y empresarial - a pesar de la resistencia de varios académicos-.

Las universidades latinoamericanas, sostiene Vessuri, deben apostar al desarrollo de investigaciones locales mediante una correcta articulación entre la docencia y la investigación universitarias ${ }^{2}$ y un nuevo contrato social entre la universidad, el Estado y el sector productivo. Según la autora, este nuevo consenso debería contemplar los siguientes aspectos:

- obteneruncompromisodelEstadoydelaindustriaparasostenerlainvestigaciónyeldesarrollo, pues solo podrán lograrse resultados efectivos en un ambiente estable y favorable;

2 Binomio que, según Vessuri, no ha tenido una relación virtuosa en la universidad de América Latina 
- prestar más atención a la investigación en el proceso de planificación universitaria y reforzar el papel de los comités de investigación en las universidades;

- atraer y mantener científicos e investigadores de calidad activos en sus campos, ofreciéndoles condiciones favorables de trabajo y apoyo financiero para estudiantes avanzados;

- reorganizar y mejorar las instalaciones para hacer posible la transformación requerida en las plataformas tecnológicas de la investigación y docencia;

- asegurar una buena coordinación con una cantidad suficiente de instituciones de educación superior y de investigación y con laboratorios industriales para la adquisición de equipos costosos, y asegurar una creciente movilidad del personal académico y de los estudiantes de postgrado;

- tratar de lograr una relación más equilibrada entre docencia e investigación, reforzando gradualmente ambos componentes;

- atraer la colaboración internacional para proyectos de investigación y desarrollo bien definidos que permitan optimizar las capacidades de investigación locales;

- promocionar centros de investigación de excelencia en o cerca de las instituciones de educación superior, en cooperación con las agencias regionales e internacionales en áreas estratégicas focalizadas, $\mathrm{y}$

- promover la comprensión del papel de la ciencia básica para el desarrollo y hacer que la investigación se vincule más estrechamente con las necesidades de la economía, el ambiente y la sociedad (Vessuri, H., 1997:157).

Estas exigencias suponen una transformación importante en el modo en que es concebida actualmente la universidad latinoamericana. No cabe dudas de que la organización tradicional de la universidad ya no logra dar cuenta de las nuevas exigencias del entorno, motivo por el cual es inminente una transformación hacia una universidad "mejor" articulada con el contexto económico y social. Si bien desde la década de los ochenta se han realizado modificaciones en la universidad latinoamericana que apuestan a esta transformación, analizar las experiencias de otros países y regiones puede ser útil para el diseño de estrategias exitosas. No se trata de copiar lo hecho por otros sino aprender de las demás experiencias y no cometer los mismos errores.

Es nuestra convicción que el análisis de las ciencias sociales y de las humanidades, en general, y de la filosofía, en particular, es clave para la consecución de sociedades más justas, con una mejor cohesión social que permita que todos los ciudadanos puedan ser reconocidos como sujetos plenos de derechos. CastroMartínez y Sutz señalan al respecto:

\footnotetext{
Será preciso, también, ayudar a los gobiernos a diseñar e implementar políticas de fomento de la ciencia y la innovación más adaptadas a sus contextos y con visión de futuro [...] que tengan en cuenta las demandas de los agentes sociales y las condiciones del contexto iberoamericano. Para ello, son de gran interés las redes de organismos nacionales de ciencia y tecnología, porque favorecen el intercambio de experiencias. Pero también la región debe propiciar el uso de las herramientas de evaluación comparativa ya utilizadas en otras partes del mundo, así como fortalecer los instrumentos que proporcionan información relevante para ello, como los observatorios. El objetivo es disponer de mecanismos para evaluar el desempeño de las organizaciones y los logros de las políticas e iniciativas emprendidas para, llegado el caso, poder reconducirlas (Ídem).
}

Esta perspectiva nos permite pensar en una universidad integrada y parte del sistema social más amplio en el que se desarrolla. Entendida de este modo, la universidad, sus investigaciones y resultados crecen conforme al crecimiento de los demás sectores sociales asociados a esta. Ya no es posible pensar esta institución como la cuna de la cultura de expertos, que extienden su conocimiento al ciudadano lego que recibe apaciblemente el conocimiento producido por una elite a la que desconoce. En cambio, la universidad del siglo 
XXI, la universidad de nuestro días es y debe ser considerada como pieza clave en el desarrollo y crecimiento del país. No se trata de una visión romántica de la universidad, sino más bien, una visión pragmática de ésta. En las sociedades de conocimiento, los sectores sociales poseen demandas y necesidades que los distintos grupos de investigación universitarios pueden satisfacer. De modo tal que el beneficio es doble, por una parte los sectores reciben solución a sus problemas y por otro los profesionales logran ocuparse en investigaciones cuyos resultados de una utilidad asegurada. En la actualidad, lejos ha quedado la universidad exclusivamente dedicada a la formación de profesionales ajenos a la labor de investigación y vinculo social.

\section{Una alternativa para la transformación de la universidad latinoamericana}

A partir de la reforma de Humboldt en 1809 en la Universidad de Berlín, la investigación se incorpora a la enseñanza universitaria. Durante el siglo xix, esta incorporación se replica en el resto de Europa y se extiende a Estados Unidos, mientras que en el siglo xx, como resultado del incremento de la responsabilidad de la universidad en el desarrollo económico y en la promoción de investigaciones funcionales a su contexto, se incorpora una tercera misión universitaria. Tal como lo sostiene Etzkowitz, la universidad pasa a tener la obligación de atender el desarrollo social y económico (Etzkowitz, H., 1998: 823). Para responder a esta nueva exigencia, surgen nuevos modelos de universidades como universidades de excelencia ${ }^{3}$, grupos de interés ${ }^{4}$, universidades empresariales y gerenciales ${ }^{5}$ y universidades de educación superior y de mercado ${ }^{6}$. Por ello, mientras la enseñanza y la investigación son la primera y segunda misión de la universidad, respectivamente, la colaboración con el desarrollo económico y social se constituye como la tercera misión de la universidad. El eje central de esta tercera misión es, tal como lo sostiene Xavier Testar, la transferencia de conocimiento y tecnología (Testar, X., 2012: 04).

La particularidad de la tercera misión de la universidad es que supone y requiere la consecución de las otras misiones. Es decir, la transferencia de conocimiento no puede ser considerada de modo aislado y descontextualizado de las demás funciones, ya que la transferencia de conocimiento y tecnología es el resultado de la expansión de la enseñanza y de la investigación. Para que haya transferencia, resulta necesario contar con un conocimiento producido por los investigadores de la universidad, y este conocimiento, además, tiene que contar con la posibilidad de ser transferido. Al mismo tiempo, el carácter formativo de la universidad es lo que garantiza la continuidad de las comunidades de expertos en todos los campos disciplinares. De este modo, podemos decir que la transferencia de conocimiento es resultado del éxito de la universidad en el cumplimiento de sus funciones tradicionales. Por lo que, en la medida en que se incremente la cantidad de conocimiento producido dentro de la universidad y en tanto se promueva una mejora en la calidad de las investigaciones y sus productos, se obtendrá una mejora en la cantidad y calidad de las transferencias realizadas de modo exitoso ${ }^{7}$. La tercera misión no solo es resultado del logro de las dos misiones anteriores, sino que además permite establecer canales de comunicación entre la universidad y el medio social en la que se encuentra. Según Arias y Aristizábal, la transferencia de conocimiento puede realizarse hacia el Estado, la industria o la comunidad a través de distintos canales y sus resultados pueden impactar en la sociedad, en la economía o a nivel epistémico dependiendo de quiénes sean los receptores del conocimiento transferido (Arias, J.; Aristizábal, B., 2011). Así, los canales a través de los que la transferencia tiene lugar dependerán de los destinatarios finales del conocimiento transferido. Estos canales pueden ser publicaciones de difusión o de divulgación, tecnologías, procesos materiales, know-how, innovación o habilidades.

\footnotetext{
Ver por ejemplo Readings, B., 1996

Ver, por ejemplo, Neave, G., 2000.

Ver, por ejemplo, Slaughter, S.; Rhoades, G., 2004.

Ver, por ejemplo, Rubiralta, M., 2004.

Si bien el concepto de transferencia tradicionalmente ha sido vinculado a la tecnología, a partir de la década de los noventa este concepto comienza a ser utilizado en un sentido más amplio. Desde entonces, al hablar de transferencia se hace referencia a la posibilidad de transferir una tecnología, el conocimiento asociado a esta tecnología o simplemente al conocimiento teórico producido en cualquier área de especialización: ciencias naturales, sociales o humanísticas.
} 
En suma, enseñanza, investigación y transferencia son los pilares de la universidad contemporánea. Por lo que, los docentes-investigadores no solo tienen la responsabilidad de formar a los futuros científicos, sino que además deben promover el desarrollo de conocimiento nuevo y socialmente útil a través de grupos de investigación. Tal como señalamos anteriormente, estas tres funciones de la universidad se vinculan a tal punto que la excelencia de una universidad en la tercera misión coincide con la excelencia en la consecución de las dos misiones restantes (Solé, F,; Berbegal, J., en Testar, X., 2012: 23). Por este motivo, la transferencia de conocimiento no se limita al conocimiento producido en el ámbito de las ciencias naturales, sino que supone que todos los campos disciplinares pueden atender distintas demandas sociales y otorgar los resultados pertinentes según las exigencias. Por lo tanto, si bien la universidad continúa siendo la institución por excelencia dedicada al cultivo de la cultura de expertos, también pasó a ser la principal proveedora de recursos profesionales e infraestructura epistémica para el desarrollo de sociedades democráticas (Emmeche, C., 2015). Logrando de este modo un correcto tránsito hacia una sociedad de conocimiento.

Pero, no hay un único modo en el cual realizar la transferencia sino que esta puede adquirir dos formas distintas: como un proceso lineal o bien como un proceso interactivo. Entender la transferencia de conocimiento científico-tecnológico desde la perspectiva lineal supone adoptar los supuestos sobre los que se funda el informe de Vannevar Bush Science: the endless frontier, de 1945. Según éste la inversión en investigación científica durante los períodos de paz y el apoyo económico a la universidad (entendida como el principal centro de desarrollo de conocimiento nuevo) son imprescindibles para el desarrollo y crecimiento de la ciencia básica. Este tipo de inversiones contribuye al crecimiento de la ciencia aplicada y al desarrollo de nuevas tecnologías. Según esta concepción, la ciencia aplicada es resultado del desarrollo de conocimiento básico, por lo que invertir en el crecimiento de la ciencia básica es estimular el desarrollo de la ciencia aplicada. De forma tal que la transferencia de conocimiento científico-tecnológico sucede de modo a-problemático y como producto del desarrollo de la ciencia aplicada.

Por otra parte, el modelo interactivo surge como alternativa al modelo lineal, y sostiene que la transferencia se caracteriza por contar con múltiples interacciones entre las distintas instituciones que la componen. Como resultado de estas interacciones, cada institución y agente involucrado en la transferencia recibe algo a cambio, lo que genera una retroalimentación entre cada una de las partes (Barge Gil, A., et al., 2006: 14). Esto permite comprender por qué ante un incremento de los fondos destinados al desarrollo del conocimiento científico-tecnológico se espera un incremento en la cantidad de resultados útiles para las demás partes.

Más allá de las modificaciones que propone el modelo interactivo respecto de cómo interactúan las instituciones, es importante subrayar que este modelo afirma la importancia de que los demás sectores sociales contribuyan al desarrollo de investigaciones en la universidad. De hecho, la implementación del modelo interactivo en las sociedades contemporáneas permitió incrementar la financiación pública en materia de investigación universitaria. Al mismo tiempo, el incremento en la cantidad de resultados socialmente útiles, producto de las investigaciones universitarias, permitió una valoración positiva en el sector económico de las universidades como institución auxiliar en el desarrollo económico y social. Por lo que la transferencia de conocimiento científico-tecnológico es, ante todo, un mecanismo de comunicación entre agentes y entre instituciones; se trata de un tipo complejo de comunicación que se ajusta al modelo interactivo y cuyo dinamismo hace del sistema un complejo evolutivo.

La complejidad del sistema constituido a partir de la transferencia como tercera misión de la universidad esta dada por la variedad de agentes y factores que intervienen en dicho proceso. Según Bozeman, estos factores y agentes son los siguientes:

Objeto a transferir: este objeto podrá ser más o menos complejo, más o menos tácito o explícito.

Productores de conocimiento: refiere al científico en tanto proveedor de conocimiento y a la institución donde se lleva a cabo la investigación científica; la institución, además de otorgar la infraestructura material para poder hacer ciencia, establece las políticas científicas que regulan la actividad científica. 
Medios de transferencia: las características de los medios de transferencia son esenciales para que esta sea efectiva; estos medios pueden ser formales o informales (licencias de patentes, consultorías, spin-offs, intercambio de personal, etc.), dependiendo de los objetivos de la transferencia, tal como veremos más adelante.

Destinatarios del conocimiento: las características de los destinatarios en cuanto a la capacidad de absorción del nuevo conocimiento, recursos y localización son cruciales para lograr una transferencia exitosa.

Entorno de la demanda: son factores que se relacionan con las necesidades existentes en el entorno socio-económico-cultural del objeto transferido. Este factor determina la posible relación entre el usuario y el conocimiento a transferir (Boezman, B., 2000).

Tal como puede inferirse, la transferencia de conocimiento es, efectivamente, "un proceso de interacción social orientado hacia la producción y circulación de conocimiento" (Barge Gil, A. et al., 2006: 15) El entorno en el que se desarrolla la transferencia de conocimiento es crucial, por lo cual considerar el contexto social en el que tiene lugar es indispensable a la hora de su comprensión.

Al igual que en el sistema de comunicación de la ciencia, el modo que adopta la transferencia está dado por el receptor hacia quien esta se orienta. Según Upstill y Symington (Upstill \& Symington, 2002), hay tres modos de transferencia: comercial, no-comercial y de creación de nuevas empresas. La transferencia comercial de conocimiento está estrechamente vinculada con el sector productivo, donde el trabajo conjunto entre la comunidad de expertos y la industria, el agro, el turismo y demás sectores productivos puede llevarse a cabo mediante consultorías, investigación conjunta, licencias, productos desarrollados durante las investigaciones o capacitaciones. Las consultorías son casos particulares de transferencia comercial en los que la parte interesada (el Estado o el sector productivo o social en general) acude a la universidad para la orientación sobre algún problema puntual a resolver. La investigación conjunta, por su parte, suele llevarse a cabo dentro de la universidad con fondos públicos o privados específicamente destinados y garantizados por contratos de ejecución. En este caso, los productos de la investigación científica pueden ser utilizados por la parte interesada adquiriendo las licencias correspondientes. Este tipo de transferencia puede ser entendida como el modo tradicional de transferencia de tecnología, y consiste en la explotación de la propiedad intelectual producida en el interior de la universidad (Bayona Sáez, C.; González Eransus, R. 2010: 14). Finalmente, la transferencia de conocimiento en modo de capacitación es, quizás, la más popular en la industria; no se contrata personal nuevo sino que se capacita y actualiza el personal estable de la empresa en consideración. El modo de transferencia no-comercial refiere básicamente a la difusión y divulgación del conocimiento producido; no hay un interés comercial entre las partes involucradas sino que el único elemento vinculante entre estas es la pieza de comunicación. Por último, la creación de empresas, o de Spin-off universitarias, es el modo más específico de los tres modos de transferencia y refiere a la explotación de conocimiento producido mediante la consolidación de parques tecnológicos e incubadoras, entre otras iniciativas. Los parques tecnológicos, las redes de empresas y los polos industriales en este caso adquieren gran relevancia, ya que contribuyen a la movilidad de personas, tecnología y conocimiento.

Vale señalar que, independientemente del modo que se adopte, la transferencia se presenta como un eslabón intermedio que permite la vinculación entre los procesos que se dan, por una parte en el interior de la universidad y, por otra parte en los diversos sectores sociales. Por otra parte, Holi señala que el conocimiento producido puede ser transferido hacia otros sectores o instituciones, tanto comerciales como no comerciales, como pequeñas empresas, industrias, grupos culturales, entre otros. Asimismo, la transferencia puede ocurrir en ambas direcciones entre las instituciones involucradas (Holi, M., 2008). Factores externos a la transferencia de conocimiento, tales como la situación económica general, las fuerzas del mercado, la competencia con la industria y las pretensiones de los consumidores, pueden alterar el modo en que se da la transferencia y repercutir en la calidad del proceso. Según éste autor, de una misma investigación científica pueden obtenerse diversos resultados, es decir, conocimiento nuevo, que se traduce en publicaciones, procesos, materiales, tecnologías, know-how, innovación y herramientas. Estos resultados, a su vez, pueden ser transferidos mediante distintas actividades: de cooperación, consultorías, contratos de investigación, licencias o formación, tal como lo vimos anteriormente. La transferencia del conocimiento producido a través de estas 
actividades tiene, según el esquema, un impacto económico mediado por una actividad económica particular. Ello supone que la transferencia impacta en la actividad económica al contribuir con la sociedad, con el gobierno, con empresas comerciales y otros, y repercute en la economía mediante la creación de empleos o del desarrollo de nuevos productos y servicios, entre otros. De allí que no es posible identificar un solo resultado de la transferencia de conocimiento.

Por lo tanto, la complejidad del procesos de transferencia no solo recae en los modos en que esta puede darse, sus resultados también pueden ser variados y con distintos niveles de complejidad. Según Holi, la transferencia de conocimiento puede tener impacto económico lo que muestra una clara relación entre la investigación universitaria y el impacto económico en la sociedad en la que se encuentra. Se trata de una relación compleja y no lineal en la que intervienen diversos factores. Los resultados de la transferencia de conocimiento no tienen una influencia directa sobre el mercado sino que se da a través de los actores que realmente actúan en el mercado generando un impacto económico. Tomando como referencia este modelo, Holi sostiene que el impacto económico y social de la transferencia de conocimiento puede presentarse de dos modos: como impacto bruto (gross impact) o como impacto neto (net impact) (Ídem: 25). Tanto el impacto bruto como el impacto neto son resultado de la actividad de transferencia de conocimiento como consecuencia de demandas puntuales hacia la universidad. Mientras que el impacto bruto es definido por Holi como el total de las consecuencias efectivas producto de la transferencia, el impacto neto es el impacto bruto menos una estimación de lo que hubiera ocurrido si la transferencia no hubiera tenido lugar. Dado que el impacto neto es difícil de calcular, la universidad debería evaluar sus actividades de transferencia sobre la base de los resultados del impacto bruto.

Si bien habitualmente la atención está puesta en la medición de la cantidad de conocimiento transferido, Holi sostiene que también es importante evaluar la calidad de estas transferencias. La medición de la calidad de estos procesos puede ser un buen indicador del éxito e impacto de la transferencia de conocimiento desde la universidad. Mientras que la cantidad está vinculada al conocimiento efectivamente transferido, la calidad estaría vinculada a la eficacia del proceso de transferencia en los distintos modos posibles en los que puede configurarse. Por eso, tanto la cantidad como la calidad deberían ser variables a considerar a la hora de estudiar procesos de transferencia de conocimiento. Pero, dada la complejidad del proceso que permite la canalización de una demanda desde un sector social hasta su solución una vez obtenido el conocimiento necesario, son varios los factores que intervienen en la transferencia y que pueden alterar tanto la calidad como la cantidad del proceso.

Considerando el resultado comercial de la transferencia, Bayona y González señalan que la transferencia de conocimiento otorga una ventaja competitiva a las empresas e industrias que la practican, y es una alternativa económica más para la creación de conocimiento nuevo en las universidades (Bayona Sáez, C.; González Eransus, 2010: 14). En el proceso de transferencia de conocimiento los beneficios y utilidad del conocimiento pueden ser simultáneos a todas las partes involucradas. Ello supone que tanto el proveedor de conocimiento como el receptor pueden utilizar al mismo tiempo el conocimiento producido. Según estas autoras, la frecuencia y profundidad de los canales de relación interpersonal, el grado de semejanza entre los interlocutores, la pérdida del conocimiento luego de producida la transferencia, el nivel de conocimiento de la organización por parte de sus integrantes (autoconocimiento) y la divergencia entre los intereses individuales y las metas de la organización son factores que permiten u obstaculizan la transferencia de conocimiento.

De este modo, la universidad, el estado y los diversos sectores sociales (productivos, culturales, etc.) pasan a ser los ejes de un mismo mecanismo. El modo en que estas instituciones se relacionan y los mecanismos que emergen en su intersección son objeto de diversas teorías. En la práctica surgen modelos de transferencia que difirieren sensiblemente según en qué institución se pone el acento. En términos de Gibbons et al., por ejemplo, podríamos decir que la transferencia de conocimiento conduce a la emergencia de un nuevo modo de producción de conocimiento que se ajusta a las condiciones y necesidades del contexto en el que la investigación es llevada a cabo. 


\section{Una transformación necesaria}

A lo largo del último siglo, la universidad debió hacer frente a una nueva demanda social. Durante este periodo, el contexto social y político no solo exige a la universidad más y nuevos conocimientos, sino que, además, la sociedad se ha vuelto más exigente en cuanto a la calidad y alcance del conocimiento producido. El nuevo contexto económico-social se caracteriza por el avance de la sociedad de conocimiento y la consolidación de las economías de conocimiento que establecen la producción de conocimiento como requisito para el desarrollo de la economía local y un incremento en la competitividad regional y mundial. En este nuevo entramado el desarrollo de conocimiento nuevo producido en las universidades, entonces, presenta un interés social, económico y, por consiguiente, político. Esta consideración no supone una concepción "romántica" del rol de la universidad en las sociedades contemporáneas, por el contrario, implica entender la universidad como una institución que se ha visto obligada a transformarse como resultado de nuevas dinámicas sociales. La más significativa de estas transformaciones, creemos, es la incorporación de la transferencia de conocimiento científico-tecnológico como tercera misión de la universidad contemporánea.

Pero, la consolidación de la transferencia de conocimiento como tercera misión de la universidad supone un largo proceso adaptativo de la universidad y una reorganización de las instituciones con las que interactúa (agrupadas en el sector productivo, la industria, el Estado y demás instituciones sociales). Según lo analizado, la transferencia de conocimiento se presenta como un sistema extremadamente complejo de interacción entre diversos agentes e instituciones, y con un estrecho vínculo entre el sistema de innovación y el contexto general en el que se desarrolla. Esto dificulta elaborar un modelo normativo de transferencia de conocimiento común a todas las sociedades, pues supone la consideración de las variables en juego en cada una de las instituciones. Dificultad que se incrementa en los países con problemas de distribución de la riqueza y con acceso desigual al conocimiento científico-tecnológico. Sin embrago, es posible acudir a algunas de las experiencias de los países desarrollados, no para imitarlos sino para hallaran el camino hacia una universidad socialmente situada.

Por lo tanto, al considerar la universidad como la proveedora de conocimiento nuevo y según las características presentadas sobre la transferencia como tercera misión de la universidad, podemos sostener lo siguiente:

La revolución sucedida en el interior de la universidad muestra la emergencia de nuevo modo de producción de conocimiento, similar al propuesto por Gibbons en cuanto a la capitalización y comercialización del conocimiento científico.

La investigación científica se convirtió en una actividad permeable al contexto en el que se desarrolla, en el sentido en que atiende las demandas y orientaciones que el contexto propone.

El contexto en el que se desarrolla la actividad científica está dado por el contexto social-económicocultural, tanto nacional como regional.

Dado lo anterior, la heterogeneidad del contexto social local y regional debe ser considerado a la hora de elaborar políticas y estructuras de desarrollo e innovación.

Según otras experiencias, el crecimiento de la actividad científica conduce al crecimiento económico y al bienestar social (coevolución del sistema) siempre y cuando se garanticen los medios para que todos los ciudadanos puedan acceder a estos (ciudadanía).

El incremento en la cantidad de resultados científicos volcados a la sociedad no puede darse de modo aislado sino que debe ir acompañado de un incremento en la cultura científica.

El incremento de la cultura científica contribuye a la comunicación de la ciencia, a la deliberación (y su consecuente valoración) social de la ciencia y sus resultados mediante la disminución de la idealización de la actividad científica. Lo que colabora con alcanzar mejores niveles en cuanto a cohesión social.

La disminución de la idealización de la actividad científica contribuye a un incremento en la valoración positiva del rol social del científico y la ciencia. 
El incremento en la valoración positiva del rol social del científico conduce a un incremento en la cantidad de interesados en llevar adelante la profesión científica.

Consiguientemente, creemos que la tercera misión de la universidad conduce a un nuevo modo de producción de conocimiento caracterizado por una estructura dinámica de comunicación entre las instituciones involucradas, tal como lo propone Etzkowitz con el modelo de triple hélice. Es por esto que desde nuestro punto de vista si bien la transferencia de conocimiento puede ser considerada como un medio de comunicación de la ciencia, en tanto supone cambios cognitivos en los individuos; colabora también con la consecución de sociedades más justas y equitativas. Paralelamente, los grupos de investigación universitarios logran desarrollar conocimiento nuevo con utilidad asegurada, incrementando los niveles de empleabilidad de sus egresados. Desde el punto de vista país, deja de ser necesario comprar respuestas y paquetes tecnológicos a instituciones extranjeras. Por lo que, la transferencia de conocimiento científico-tecnológico de la universidad, se convierte en un diseño de gestión del conocimiento que podría ser útil para el diseño de estrategias de crecimiento en Iberoamérica y en América Latina en particular.

En suma, el recorrido aquí planteado procura mostrar que las transformaciones que supone incorporar la transferencia como tercera misión de la universidad no pueden sucederse solo en el interior de esta institución. Muy por el contrario, es necesario elaborar políticas de ciencia y tecnología a mediano y largo plazo, articuladas con otras políticas económicas y sociales, tanto locales como regionales. Se trata de quitarnos las antiguas vestiduras y optar por un modelo de universidad atento a las demandas de los diversos sectores inmediatos de la sociedad en la que se desarrolla. Se trata, en efecto, de una transformación más que necesaria.

\section{Bibliografía}

ALBORNOZ, M., López Cerezo, J. A. (ed.) (2012) Ciencia, Tecnología y Universidad en Iberoamérica Buenos Aires: Eudeba (E-book)

ARIAS PÉREZ, J.; Aristizábal, B. (2011). “Transferencia de conocimiento orientada a la innovación social en la relación ciencia-tecnología y sociedad”. En Revista científica Pensamiento y gestión (n 31), pp. 137-166.

BARGE GIL, A.; Modrego Rico, A. y L. Santamaría Sánchez (2006). El proceso de transferencia tecnológica UniversidadEmpresa. Madrid: Fundación Empresa Ciencia.

BAYONA SÁEZ, C.; González Eransus, R. (2010). La transferencia de conocimiento en la Universidad Pública de Navarra. Una visión desde la empresa y desde el ámbito universitario. Navarra: Universidad Pública de Navarra.

BOZEMAN, B. (2000). “Technology transfer and public policy: a review of research and theory”. En Research Policy, vol. 29 (issues 4-5), pp. 627-655.

EMMECHE, C. (2015). “Universities as Marketplaces”. En Science \& Education, vol. 24 (issue 7), pp. 1047- 1054.

ETZKOWITZ, H.; Leydesorff, L. (1997) Universities in the global economy: A triple helix of university-industry-government realtions Londres: Cassell Academic

ETZKOWITZ, H., (1998). "The norm of entrepreneurial science: cognitive effects of the new university-industry linkage”. En Research Policy, vol. 27 (issue 8), pp. 823-833.

ETZKOWITZ, H.; Leydesdorff, L. (2000). "The dynamics of innovation: from a National System and 'Model 2' to a Triple Helix of University-industry-government relations”. En Research Policy, vol. 29 (issue 2), pp. 109-123.

ETZKOWITZ, H (2008). The Triple Helix. University-Industry-Government. Innovation in Action. U.S.A: Routledge Taylor \& Francis Group.

ETZKOWITZ, H. (2010). A company of their own: entrepreneurial scientist and the capitalization of knowledge. En Viale, R; Etzkowitz (ed.), The capitalization of knowledge. A triple helix of University-Industry-Government. USA: Edward Elgar Publishing.

ETZKOWITZ, H. (2015). “Making Humanities town: knowledge-infused clusters, civic entrepreneurship and civil 
society in local innovation systems”. En Triple Helix, vol. 2 (issue 1).

GIBBONS, M et al. [1994] (1997). La nueva producción de conocimiento. La dinámica de la ciencia y la investigación en las ciencias contemporáneas. Barcelona: Ediciones Pomares-Corredor, S.A.

HOLI, M. (2008). Metrics for the evaluation of knowledge transfer activities at universities. Cambridge: Library House.

READINGS, B. (1996) The university in ruins Londres: Harvard University Press

RUBIRALTA, M. (2004) Transferencia a las empresas de la investigación universitaria Descripción de modelos europeos Madrid: Fundación COTEC

SLAUGHTER, S.; Rhoades, G. (2004). Academic capitalism and the new economy markets, state and higher education Baltimore: The Jhon Hopking University Press

TESTAR, X. (2012). La transferencia de tecnología y conocimiento universidad-empresa en España: estado actual, retos y oportunidades. Barcelona: Colección documentos CYD.

UPSTILL, G.; Symington, D. (2002). “Technology transfer and the creation of companies: the CSIRO experience”. En R\&D Management, vol. 32, (issue 3), pp.233-239.

VESSURI, H. (1997) "Investigación y desarrollo en la universidad latinoamericana" en Revista Mexicana de Sociologia, vol. 59, No 3, pp. 131-160

VESSURI, H. (2014) “Cambios en las ciencias ante el impacto de la globalización” en Revista de estudios sociales, No 50, pp. $167-173$

ZIMAN, J. (2003) “Ciencia y sociedad civil” Isegoria, vol. 28, pág. 5-17 